QUADERNS DE FILOSOFIA VOL. IV NÚM. I (20I7): I I-23

eISSN: 234I-3042 DOI: IO.7203/QFIA.4.I.IO208

Javier Gracia Calandín

Universitat de València

\title{
Repensando la innovación educativa en y desde la filosofía. Estudio introductorio ${ }^{1}$
}

(Rethinking Educational Innovation in and from Philosophy. Introductory Study)

Resumen: La innovación educativa supone un reto para la filosofía. Un reto porque nos lleva a replantear el magisterio de la filosofía a la luz de los últimos avances en la investigación educativa. Pero también porque nos hace volver con energías renovadas a las fuentes originales de la actitud filosófica de vigilancia y de búsqueda sin término del conocimiento y de la verdad. En estas líneas, en primer lugar, analizamos filosóficamente la relevancia que ha cobrado la innovación en el ámbito educativo y proponemos una visión humanista (no reductiva a las nuevas tecnologías) para entender la innovación educativa con especial significación para la labor educativa de la filosofía. En segundo lugar, invitamos a la lectura de todos y cada uno de los artículos seleccionados en este volumen monográfico.

Abstract: Educational innovation is a challenge for philosophy. This is a challenge because it leads us to rethink the teaching of philosophy in the light of the latest advances in educational research. But also because it makes us return with renewed energy to the original sources of the philosophical attitude of vigilance and unfinished search of knowledge and truth. In this paper, firstly, I analyse philosophically the relevance of innovation in the educational field and propose a humanistic vision (not reductive to new technologies) to understand educational innovation with special

${ }^{1}$ Este trabajo se inscribe dentro del proyecto con referencia FFI2016-76753-C2-1-P financiado por el MINECO y del proyecto de innovación educativa con referencia UV-SFPIE_RMD 15-377379. 
significance for the educational task of philosophy. Secondly, I invite you to read each and every one of the selected articles in this monographic volume.

Palabras clave: innovación educativa, filosofía, formación humana, visión humanista. Keywords: educational innovation, philosophy, human formation, humanist vision.

\section{DESBROZANDO EL CONCEPTO DE INNOVACIÓN EDUCATIVA DESDE LA FILOSOFÍA}

$\mathrm{H}$ ABLAR DE INNOVACIÓN EDUCATIVA constituye uno de los temas más recurrentes en las políticas educativas, sin embargo, gran parte de estas alusiones están lastradas por una falta de profundización. De modo muy significativo la expresión "innovación educativa” ha cobrado en los últimos tiempos una presencia muy notable en los ámbitos académicos y ya se cuentan muchas las iniciativas relacionadas con la innovación educativa tales como congresos, jornadas, proyectos y grupos de trabajo en diferentes niveles, ámbitos y especialidades. ${ }^{2}$

Desde la reforma del Espacio Europeo de Educación superior se ha puesto el énfasis en una reforma de la educación que incorpore nuevas metodologías docentes, incidiendo en un seguimiento más personalizado y significativo del aprendizaje del alumnado. A este respecto las políticas educativas han enfatizado que la educación actual ha de ser una educación innovadora (CARBONELL 200 i; González 2009 y SÁnchez 20I4). Como reflejo de esto se han institucionalizado los servicios que promueven la innovación entre sus docentes. ${ }^{3}$

\subsection{Más allá del imperativo legal}

La importancia que las administraciones conceden a la innovación educativa ha hecho que uno de los principios y fines de la educación recogidos

${ }^{2}$ Un buen repositorio de "Buenas prácticas en innovación" promovido por el Ministerio de Educación, Cultura y Deporte puede encontrarse en http://138.4.83.162/organiza/buscador_ buenaspracticas/. Por lo que respecta a la filosofía, del 5 al 7 de noviembre de 2014 la Facultad de Filosofía de la Universidad Complutense de Madrid organizó las Jornadas Internacionales de Innovación Didáctica en la Enseñanza de la Filosofía y más recientemente, del 19 al 21 de octubre de 2016, tuvo lugar el II Congreso Internacional de Innovación Educativa en la Filosofía en la Facultad de Filosofía y CCEE de la Universidad de Valencia.

${ }^{3}$ Por ejemplo, en el ámbito autonómico la actual Conselleria d'Educació, Investigació, Cultura i Esport tiene destinado un espacio donde reflejar la innovación educativa (http:// www.ceice.gva.es/eva/es/innovacion.htm) [consultado 7 de abril de 2016]. Otro ejemplo lo encontramos en la Universidad de Valencia que tiene un "Servicio de Formación Permanente e Innovación educativa” (http://www.uv.es/uvweb/servicio-formacion-permanente-innovacioneducativa/es/servicio-formacion-permanente-innovacion-educativa-sfpie-1285866029030. html). [Consultado 7 de abril de 2017] 
en la Ley Orgánica de la Mejora de la Calidad Educativa (LOMCE) sea "el fomento y la promoción de la investigación, la experimentación y la innovación educativa”. El tratamiento que la actual legislación da a la innovación educativa sigue el camino marcado por las anteriores leyes educativas y recoge, en muchos puntos literalmente, lo que estableció la Ley Orgánica de Calidad de la Educación (LOCE) y la Ley Orgánica de Educación (LOE). ${ }^{4}$

Aunque no se puede desatender lo que la normativa indica, sin embargo, la significación de la innovación educativa trasciende al ordenamiento o la coyuntura de una política educativa particular. La innovación educativa incide en diversidad de aspectos que implican repensar desde la raíz la esencia propia de la educación así como las diversas formas de concretar los procesos de aprendizaje y enseñanza a partir de nuevas metodologías. Por eso una reflexión más profunda desde la filosofía acerca del reto que implica la innovación en nuestro sistema educativo ha de plantearse no solo las herramientas o procedimientos sino también el potencial formador y transformador de las nuevas metodologías.

Efectivamente, aunque tal y como recoge la Real Academia de la Lengua el término "innovación" significa "la creación y modificación de un producto y su introducción en el mercado", al añadirle el adjetivo "educativa" resulta —a mi juicio- que no estamos expuestos a asumir exclusivamente las connotaciones técnicas, sino que se abre la posibilidad de considerar un sentido menos mercantilista e instrumentalista de innovación, abogando por la introducción de nuevas metodologías que contribuyan a una más fecunda formación humana. Esta segunda acepción referida a un concepto fundamental de educación como formación humana está, de modo inequívoco, estrechamente vinculada con la visión humanista de la educación (UNESCO 2015). De este modo observamos que en los dos términos de la expresión "innovación educativa" se establece ya una interesante tensión entre la dimensión técnica e instrumental, por una parte, y la dimensión más humanista, por la otra.

\subsection{La paradoja de la innovación}

Como en otras épocas también la nuestra tiene sus palabras de modas que acaban convirtiéndose en términos fetiches. Tal es el caso del vocablo

\footnotetext{
${ }^{4}$ En el título preliminar, capitulo 1.o, "Principios y fines de la educación", en su artículo $1 \mathrm{n}$ ), considera como uno de los principios de la educación "el fomento de la promoción de la investigación, la experimentación y la innovación educativa”. En ese mismo capítulo, el artículo 10.1 se refiere a la difusión de la información, indicando que a las administraciones educativas les corresponde facilitar los intercambios de información y difusión de buenas prácticas educativas y de gestión de centros docentes, a fin de contribuir a la mejora de la calidad de la educación. Para un análisis del tratamiento de la innovación educativa en la LOE puede consultarse el detallado y prolijo Estudio sobre la innovación educativa en España (JuÁrez 201 I , 44-5).
} 
"innovación" que en nuestros días se ve expuesto a un uso inflacionario. Desde la tribuna política o el foro de empresarios hasta los ámbitos educativos o la rueda de prensa deportiva, podemos encontrar la utilización de dicho término para recubrir las propias ideas con códigos socialmente aceptados. ¿o es esto una muestra de que el propio concepto de innovación no escapa a la lógica de que solo sobrevive aquello que es introducido con éxito en el mercado? Bajo este prisma podría decirse que la primera prueba de que la innovación funciona es precisamente que ella ha devenido moneda de cambio. Pero, ¿no conduce esto precisamente a un uso inflacionario de innovación que termina dando por bueno cualquier cosa por el hecho de ser novedad?

Sin embargo, también podría hacerse la lectura contraria: que precisamente aquellas innovaciones buenas acaben siendo duraderas y, por lo tanto, conservadoras, y paralicen la innovación. $\mathrm{Y}$ es esta una flagrante paradoja porque efectivamente "las mejores innovaciones son las más duraderas en el tiempo y, por tanto, las más resistentes a posteriores innovaciones" (РАCHO 2009, 39).

Aunque este argumento pueda resultar subversivo para aquellos que preconizan un modus operandi innovador sin reflexionar en la importancia y el valor de la innovación llevada a cabo, a mi juicio, resulta fundamental para defender que la innovación puede conducir a este tipo de paradojas o incluso aporías si no va acompañada de un fin específico, en nuestro caso los propios de la educación. Es decir, si no se supera la tendencia a pensar que por el hecho de que algo sea nuevo ha de ser bueno. La novedad no tienen por qué ir siempre de la mano de la calidad educativa.

\subsection{Superar la innovofilia hodierna}

Innovar es efectivamente un signo de los tiempos modernos. Lo es porque el término innovar introduce el elemento de la novedad y ello evidencia que, frente a la tradición, desde la "modernidad" se incide en el "ahora mismo" o "este momento", deviniendo la moda uno de los principales reclamos de nuestra forma de vida moderna.

Mas desde la radicalidad que confiere la reflexión filosófica cabría preguntarse: ¿cuál es la relación que guardan entre sí la innovación y la tradición? Para esclarecer qué estatuto corresponde a la innovación y el hecho de que la novedad se transforme en valor resulta apremiante un análisis filosófico que tome en consideración el cambio de paradigma que introduce la modernidad con la llegada de una nueva cosmovisión alentada por el auge y el desarrollo de las ciencias experimentales y el poder transformador que adquiere la técnica. La traslación del antiguo provenir al moderno porvenir sintetiza la mentalidad 
de un mundo que no pone sus miras en el pasado de la tradición sino en el futuro de la innovación. De este modo se va consolidando la concepción de que es en el porvenir en el que lo mejor está por suceder. Pero, ¿̨hasta qué punto puede haber innovación sin tradición?

A esta sazón, cabría preguntarse si este sentido de innovación y de modernidad que conducen al valor de lo que está de moda no es excesivamente superficial y especialmente perjudicial para el ámbito educativo. Confundir lo novedoso con las innovaciones buenas y necesarias es sencillamente un error. Por ello hace falta ir al fondo de la cuestión de la innovación y superar la innovofilia hodierna. Pensar en el aquí y ahora sin tener en cuenta el ayer o el mañana conduce a repetir los mismos errores que en el pasado.

A mi juicio, la buena innovación educativa ha de tener en cuenta la tradición o, para ser más justo, la diversidad de tradiciones habidas (y por haber), pero sin menoscabo de las nuevas circunstancias personales y sociales. La buena innovación necesita de la reflexión y de un análisis de la naturaleza no clausurada del ser humano, de su ser pro-yecto, esto es, lanzado hacia el futuro pero desde un pasado. El ahora pierde su textura sin la urdimbre del tejido narrativo del antes y el después.

Toda enseñanza implica la transmisión de determinados saberes, mas especialmente en la filosofía la tradición constituye un elemento extraordinariamente fecundo, por ejemplo, a partir del estudio de los clásicos. Sin embargo, hay que replantearse si reconocer y apropiarse el legado del pensamiento de los grandes filósofos conduce a eliminar la innovación; o por el contrario tradición e innovación constituyen en la filosofía dos elementos enormemente fecundos cuando son adecuadamente complementados. ¿De qué modo llevar a cabo dicha complementación en la práctica docente?

\subsection{La innovación educativa desde la perspectiva humanista}

En la década de 1960 irrumpe la primera perspectiva de la innovación educativa marcada por modelos traídos de la industria, la agricultura y métodos cuantitativos. Muchos siguen entendiendo aún hoy en día que la innovación educativa solo significa el uso de las tecnologías de la comunicación y la información (TICs). Pero esta solo es la "perspectiva tecnológica" que ha venido siendo complementada por la "perspectiva cultural" y la "perspectiva sociopolítica” (JuÁrEZ 20 I I, 27-30).

La buena innovación no puede estar guiada por la lógica del mercado y por una racionalidad exclusivamente instrumental. A mi juicio el modo más técnico y tecnológico de entender la innovación educativa que sin duda está marcado por la lógica del mercado no debe acallar la cuestión de fondo del 
fin de toda educación. Pues "educar" no es solo ni principalmente instruir y mucho menos adoctrinar, por ello es apremiante que se esclarezca cuál es el planteamiento que permite desarrollar y emplear todo el potencial de las nuevas tecnologías de la información y la comunicación con fines "educativos".

Se trata efectivamente de volver a plantearse la pregunta crucial, ¿para qué innovar en educación?, ¿`cuál es el fin de la innovación educativa? ¿Es la innovación un fin de la educación (como dice, por ejemplo, la LOMCE) o es más bien la educación un fin de la innovación (Gracia y GozÁLVEZ 20I6)?

Pensar que el fin de la educación es formar profesionalmente a las personas para prepararlas para el mundo laboral fomenta un tipo de innovación al servicio de las demandas del mercado. ${ }^{5}$ Sin duda, es importante este fin y no puede ser desatendido, (¿tampoco por la filosofía?). Incluso creo que puede ser fecundo investigar de qué modo es posible diseñar desde la filosofía procedimientos novedosos para resolver problemas concretos. De hecho ya se habla de innovación en humanidades en la medida en que se transfiere conocimiento humanístico al tejido socioeconómico para hacerlo competitivo. Aparece así una transferencia en el mundo de la cultura que resulta en productos cinematográficos, discográficos, audiovisuales o editoriales; también tiene una fuerte presencia, por ejemplo, la transferencia en museos, fundaciones, medios de comunicación o empresas encargadas de la rehabilitación y conservación del patrimonio.

Con todo, ha lugar para seguir interpelándose, ¿es la profesionalización el fin primordial y más fundamental de la educación (Pring 2003)?, ¿’o es acaso y por añadidura de la innovación educativa? ¿De qué modo es posible innovar en las humanidades (CorTina 2013)? ¿No es acaso el fin primero y más fundamental de la educación (y de la innovación educativa) la formación cívica y humana de las personas? ${ }^{6} \mathrm{Si}$ asumimos esto, entonces el resto de objetivos han de estar supeditados a este. En este sentido cabe reafirmar en nuestros días la visión humanista de la educación que enfoca precisamente un modelo de desarrollo humano en toda su amplitud poniendo un énfasis especial en la cuestión normativa referida a los valores y principios éticos que deben regir la educación (UNESCO 20 I 5; Nussbaum 2010; Pring 20i4; Gracia 2017...).

${ }^{5}$ A juicio de algunos, una concepción mercantilista de la educación estaría en el trasfondo de la LOMCE; de modo más evidente en las versiones iniciales del preámbulo (SubiraTs 20 i4).

${ }^{6}$ En el artículo 26 de la Declaración Universal de los Derechos Humanos está muy claramente expresado: "La educación tendrá por objeto el pleno desarrollo de la personalidad y el fortalecimiento del respeto a los derechos humanos y a las libertades fundamentales; favorecerá la comprensión, la tolerancia y la amistad entre todas las naciones y todos los grupos étnicos y religiosos; y promoverá el desarrollo de las actividades de Naciones Unidas para el mantenimiento de la paz". Esto mismo fue casi literalmente recogido en el artículo 27 de la Constitución Española: "[l]a educación tendrá por objeto el pleno desarrollo de la personalidad humana en el respeto a los principios democráticos y a los derechos y libertades fundamentales". 
A mi juicio, la filosofía tiene mucho que decir sobre la innovación educativa. Pues más allá de aplicaciones a determinados sectores, el valor de la filosofía radica precisamente en generar y crear el humus desde el que es posible el cultivo de las personas y de los ciudadanos. A este respecto sería muy fecundo desentrañar el vínculo inextricable entre la educación y la clásica Bildung (formación). Sobre la égida de este concepto el valor de la educación nunca será sustituible por precios del mercado. Y aún con todo — podemos seguir preguntándonos- atendiendo a esta tarea prioritaria de la filosofía, ¿̇de qué modo es posible la innovación educativa en y desde la filosofía?

\section{INVITACiÓN A LA LECTURA DE LOS ARTÍCULOS DEL MONOGRÁFICO PARA REPENSAR LA INNOVACIÓN EDUCATIVA EN Y DESDE LA FILOSOFÍA}

El presente número monográfico sobre innovación educativa en la filosofía recoge tanto artículos que reflexionan filosóficamente en torno a la innovación y, más en concreto, a la innovación educativa, como otro tipo de artículos que presentan propuestas didácticas en las asignaturas de filosofía. Pensamos que ambos enfoques son complementarios y tienen cabida en este número puesto que están implicados en el ejercicio docente e investigador de la práctica filosófica. Por una parte, la indagación filosófica acerca de la génesis y los fundamentos de la innovación educativa constituyen una labor de enorme calado filosófico, que busca dar con las raíces y sentido último de la práctica innovadora. Por otra parte, la concreción de propuestas didácticas innovadoras constituye la mejor ejemplificación de que también es posible innovar en nuevas metodologías educativas en las asignaturas de la filosofía (filosofía, historia de la filosofía, ética, antropología, lógica, estética...). Resulta un valor añadido que dichas propuestas estén enriquecidas como experiencias de innovación educativa y ofrezcan pistas de cómo abordar mejor su aplicación dentro del aula.

En primer lugar, José Antonio Marín se detiene a analizar desde la reflexión filosófica la génesis del valor de la innovación y la relevancia que esta adquiere en la modernidad. Pues en la era moderna un ritmo de vida acelerado acaba por instalarse en un presente fugaz marcado por la conciencia de la finitud

\footnotetext{
${ }^{7}$ Véase el prolijo estudio de Vierhaus (I972), donde desentrańa la Bildung como "concepto pedagógico" vinculándolo al de Erziehung (educación) al menos desde 1770, en plena Ilustración. Todo lo cual nos llevaría a profundizar en autores como Herder, Goethe o Humboldt (VIERHAUS I 972, 512-21). El concepto ciceroniano de "cultura" es a esta sazón el principal referente de la formación humana lo cual expresa el quehacer más singular y propio de la filosofía. Véase Gracia (2012).
} 
y la brevedad (de tiempo) de la vida humana. Guiado por planteamientos orteguianos y heideggerianos, José Antonio Marín cuestiona la "sobrecarga" moderna de innovación y se pregunta cuál es el cometido más propio de la filosofía. De este modo, a la luz de la "hermenéutica adaptativa" de Odo Marquard, concluye que merced a la filosofía es posible una "descarga" del ímpetu de innovación que atraviesa la vida moderna y que se concreta en el cultivo de la tradición y la cultura de continuidad. Compensación que no ha de entenderse como sustitución, sino como el equilibro que el mundo moderno necesita entre el ritmo acelerado y cambiante marcado por la alianza entre la técnica y las ciencias naturales y el estudio reposado y profundo de la tradición que ofrecen las humanidades. Solo en este equilibrio es posible mantener la fecunda tensión entre el provenir y el porvenir; la experiencia y la expectativa; el "devenimiento" y el advenimiento; la tradición y la innovación.

Incidiendo en las posibilidades de encontrar un buen marco filosófico en el que asentar la innovación educativa, Alfredo Esteve apunta en el segundo de los artículos que aquí presentamos de qué forma es posible una fundamentación antropológica de la educación desde la filosofía zubiriana incidiendo en su doble dimensión tanto intelectiva como afectiva. Al hacerlo, destaca el lugar central que en ella adquiere la creatividad y la singularidad humana. El artículo analiza el surgimiento de aspectos muy vinculados con la innovación tales como la "creatividad". Considerando la educación como un proceso complejo en el que intervienen elementos cognitivos, volitivos y afectivos y en el cual la filosofía adquiere un papel protagonista, el artículo concluye con una invitación a la "pedagogía de la admiración" de Alfonso López Quintás.

En este mundo moderno de lo cambiante las nuevas tecnologías han adquirido un inequívoco protagonismo y cada día se cuentan más las actividades que realizamos a través de las tecnologías de la información y comunicación (redes sociales, gestión bancaria, consumo de oferta cultural, comercio electrónico...). Cabría preguntarse qué papel le corresponde jugar a las nuevas tecnologías de la información y la comunicación en la educación. ¿̇Ha penetrado suficientemente la tecnología en el ámbito educativo? ¿Qué tipo de rol le corresponde jugar a la filosofía y, en concreto, a la ética en un mundo cibernético? ¿Qué implicaciones tiene esto para la educación? Estas son algunas de las principales cuestiones que se plantea Carlos Rodríguez en su artículo y en el que reivindica una comprensión más profunda del fenómeno de la tecnología y que no solo sea concebida como mera adquisición de destrezas orientadas a las competencias relacionadas con la artesanía y el diseño. A este respecto cabe situar su defensa de una educación encaminada a generar una "ciberciudadanía responsable".

Frecuentemente se ha criticado la sociedad actual por ser excesivamente individualista. Esto tiene también su reflejo en el sistema educativo que pro- 
mueve aprendizajes individuales y olvida que la educación es un proceso dialógico que solo tiene lugar en la tejido de la acción comunicativa y cuyo fin es la emancipación y la humanización de los seres humanos. En este sentido, resulta apremiante indagar en nuevas metodologías y propuestas didácticas que fomenten la comunidad educativa. ¿De qué forma es posible innovar en metodologías educativas de forma que podamos fortalecer los vínculos sociales? ¿Qué ventajas ofrecen estas nuevas metodologías cooperativas para el aprendizaje?

Entre las diferentes propuestas emergentes en el ámbito escolar, destaca la metodología de las "Comunidades de aprendizaje". Partiendo de su aquilatada experiencia docente en centros de educación secundaria, universitaria, Carmen Ferrete nos presenta el potencial que ofrecen las comunidades de aprendizaje en tanto que metodología interactiva y cooperativa, que permiten que los centros educativos sirvan como instrumentos de cohesión social y como lugares que propicien la transformación social y educativa. De entre las diversas modalidades que esta metodología ofrece, la autora se centra en las comunidades de aprendizaje referidas al aula y al centro y presenta tres prácticas inclusivas: los grupos interactivos, las tertulias filosóficas y la tertulia dialógica pedagógica. La autora reconoce la adecuación de dichas metodologías para las asignaturas del departamento de filosofía y concluye destacando las valiosas ventajas que estas prácticas ofrecen en términos de trabajo en equipo, solidaridad e implicación de la comunidad en la educación.

La importancia de la comunidad también ha sido destacada en el ámbito de la innovación educativa en las llamadas "comunidades de investigación". Habida cuenta del modelo clásico de lectura individual y solitaria de un libro que concluye con la realización de un trabajo con preguntas prefijadas por el profesor, ¿de qué forma es posible innovar?, ¿cómo hacer del aula una comunidad en la que los estudiantes indaguen juntos y aprendan los unos de los otros? Tomando como texto de referencia la célebre obra de Primo Levi Si esto es un hombre, Ana Lucía Batalla presenta la realización del seminario de lectura como una experiencia educativa innovadora.

Por otro lado, hoy en día ocupan un lugar muy destacado las diferentes propuestas educativas que inciden en un tipo de aprendizaje que sea significativo a la vez que gratificante. Abundan en la idea de que aprender puede ser algo divertido y de que todo lo que se aprende jugando permanece más sólidamente en los conocimientos del niño. Esto plantea algunas cuestiones filosóficas interesantes tanto por el fondo como por la forma; tanto por lo que se refiere a la reflexión en torno a la relación entre educación y juego, como por lo que se refiere a la idoneidad de propuestas didácticas para la enseñanza y aprendizaje de la filosofía. ¿Debe ser la experiencia educativa gratificante además de significativa? ¿De qué forma es posible hacer del aprendizaje y la enseñanza de 
la filosofía una experiencia gratificante? Carla Carreras en "Del homo ludens a la gamificación" nos presenta un análisis del concepto de gamificación y sondea las posibilidades que este ofrece en la enseñanza de la filosofía.

En su artículo "Taller de significados inéditos", Luis Calero presenta una propuesta muy innovadora en la didáctica de la filosofía consistente en generar nuevos significados a partir de la redefinición de conceptos siguiendo unas sencillas reglas como la de fusión o separación. El autor no solo nos ofrece una descripción general de esta metodología, sino que recoge una breve guía didáctica con ejemplos que sin duda será muy útil para el profesorado que desee emplearla en clase. Esta innovadora práctica sin duda destaca por su potencial creativo y también lúdico.

Mas las posibilidades de innovar educativamente en la filosofía no se restringen a las aulas. Más bien habría que preguntarse si es posible encontrar otros cauces de educación (formal o no formal) capaces de hacer visible el patrimonio cultural y que tengan una especial significación en general para la filosofía y en particular para la estética. ¿En qué medida es posible aprovechar el espacio que ofrecen los museos para fomentar un diálogo filosófico entre los visitantes y las obras? ¿Cómo hacer de los espacios museísticos una institución verdaderamente educativa que contribuya a una reflexión filosófica? En su artículo "Explorando las posibilidades de la filosofía en el campo de la educación patrimonial", Jordi Arcos nos presenta la "museografía didáctica" como innovación educativa en la filosofía en tanto que permite complementar el pensamiento de los filósofos con su contexto biográfico.

Todos los artículos son una variada muestra de que la innovación educativa es una realidad que de múltiples formas va cobrando mayor presencia en el mundo académico. Junto con Pedro Jesús Teruel como editor coordinador de este número agradezco al Consejo editorial de la revista Quaderns de filosofia la acogida de nuestra iniciativa y la oportunidad que nos ha brindado de poder elaborar un monográfico sobre esta cuestión. La propuesta de profundizar en el tema de la innovación educativa en la filosofía vino avalada inicialmente por la realización del II Congreso internacional de innovación educativa en la filosofía que tuvo lugar en Valencia del 19 al 21 de octubre de 2016. Este propició el espacio idóneo para la reflexión filosófica y fue muy fecundo para la discusión en torno a diversos temas vinculados con la innovación educativa en la filosofía. Algunas de dichas propuestas ven ahora la luz en este monográfico. De forma complementaria, desde la página web de la revista Quaderns de filosofía se hizo una llamada para el envío de originales sobre la innovación educativa en la filosofía, de modo que quien quisiera (aunque no hubiese participado en dicho congreso) pudiera enviar sus originales. Agradecemos a todos los que 
presentaron sus propuestas para ser evaluadas y también a todos y cada uno de los revisores que con la garantía del anonimato de los autores realizaron sus respectivos informes de evaluación. 


\section{BIBLIOGRAFÍA}

Carbonell, J. 200i, La aventura de innovar. El cambio en la escuela, Madrid: Morata.

Cortina, A. 2013, "¿Es posible innovar en las humanidades? Los avances humanísticos mejoran la competitividad social”, El Pais, 15 de julio. http:// elpais.com/elpais/2013/07/08/opinion/1373286321_629687.html [consultado el 7 de abril de 2017].

De La Torre, S. 1997, Innovación educativa. I. El proceso de innovación, Madrid: Dykinson.

González, J. 2009, Innovación educativa y espacio europeo de educación superior, Málaga: Universidad de Málaga.

Gracia, J. 201 2, "Recreaciones filosóficas sobre la metáfora ciceroniana de cultura”, E. Casaban (ed.), XIX Congrés Valencià de Filosofia, Valencia: SFPV.

Gracia, J. 20 I7, "El fin ético-filosófico de la educación”, en P. Calvo y M. Medina, Mirades intersubjectives en al filosofia actual, Valencia: SFPV, 21-6.

Gracia, J. y GozÁlvez, V. 2016, "Justificación filosófica de la educación en valores éticos y cívicos en la educación formal. Análisis crítico de la LOMCE”, Teoria de la Educación, 28 (1): 83-103.

JuÁrez, E. M. 20 I I, "Perspectiva histórica de la innovación educativa en España”, en Ministerio de Educación, Estudio sobre la innovación educativa en España, Madrid: Secretaria General Técnica.

Nussbaum, M. 2oio, Non For Profit. Why Democracy Needs The Humanities, Princeton: Princeton University Press.

PACHO, J. 2009, "La paradoja de la innovación. Aspectos axiológicos y epistémicoculturales", en M. J. Maidagán, I. Ceberio, L. Garagalza y G. ArrizabaLAGA (eds.), Filosofía de la innovación. El papel de la creatividad en un mundo global, Madrid: Plaza y Valdés, 33-44.

Pring, R. 2003, "La polémica sobre los fines de la educación", en M. García Amilburu, Claves de la Filosofía de la educación, Madrid: Dykinson.

Pring, R. 20I4, "From Disguised Nonsense to Patent Nonsense: Thinking Philosophically", Revista española de pedagogía, 258, 72 (mayo-agosto): 24961.

SÁNCHez, A. 20 I 4, Innovación docente en los nuevos estudios universitarios, Valencia: Tirant Humanidades.

Subirats, M. 20 14, "La LOMCE: Hacia una educación antidemocrática", Revista Interuniversitaria de Formación del Profesorado, 81: 45-58.

UNESCO 2015 , Replantear la educación. ¿Hacia un bien común mundial?, París: UNESCO. 
Vierhaus, R. I974, "Bildung”, Brunner, O., Conze, W. y Koselleck, R., Geschichtliche Grundbegriffe: Historisches Lexikon zur politisch-sozialen Sprache in Deutschland., Bände 1, Stuttgart: Klett-Cotta, 508-51. 